\title{
New Results in the Calculus of Fuzzy-Valued Functions Using Mid-Point Representations
}

\author{
Luciano Stefanini $^{1(\bowtie)}{ }^{\left(\text {Laerte Sorini }^{1} \text {, and Mina Shahidi }\right.}{ }^{1,2}$ \\ 1 DESP - University of Urbino Carlo Bo, Urbino, Italy \\ \{luciano.stef anini, laerte.sorini\}@uniurb.it \\ 2 Department of Mathematics - IASBS Institute, Zanjan, Iran \\ minashahidi@iasbs.ac.ir
}

\begin{abstract}
We present new results in the calculus for fuzzy-valued functions of a single real variable. We adopt extensively the midpoint-radius representation of intervals in the real half-plane and show its usefulness in fuzzy calculus. Concepts related to convergence and limits, continuity, level-wise gH-differentiability of first and second orders have nice and useful midpoint expressions. Using mid-point representation of fuzzy-valued functions, partial orders and properties of monotonicity and convexity are discussed and analysed in detail. Periodicity is easy to represent and identify. Graphical examples and pictures accompany the presentation.
\end{abstract}

Keywords: Fuzzy-valued function - Midpoint representation · Monotonic fuzzy function · Convexity of fuzzy function · Periodic fuzzy function

\section{Introduction to Intervals and Fuzzy Numbers}

We denote by $\mathcal{K}_{C}$ the family of all bounded closed intervals in $\mathbb{R}$, i.e.,

$$
\mathcal{K}_{C}=\left\{\left[a^{-}, a^{+}\right] \mid a^{-}, a^{+} \in \mathbb{R} \text { and } a^{-} \leq a^{+}\right\} .
$$

To describe and represent basic concepts and operations for real intervals, the well-known midpoint-radius representation is very useful (see e.g. [2] and the references therein): for a given interval $A=\left[a^{-}, a^{+}\right]$, define the midpoint $\widehat{a}$ and radius $\widetilde{a}$, respectively, by

$$
\widehat{a}=\frac{a^{+}+a^{-}}{2} \text { and } \widetilde{a}=\frac{a^{+}-a^{-}}{2},
$$

so that $a^{-}=\widehat{a}-\widetilde{a}$ and $a^{+}=\widehat{a}+\widetilde{a}$. We denote an interval by $A=\left[a^{-}, a^{+}\right]$or, in midpoint notation, by $A=(\widehat{a} ; \widetilde{a})$; so

$$
\mathcal{K}_{C}=\{(\widehat{a} ; \widetilde{a}) \mid \widehat{a}, \widetilde{a} \in \mathbb{R} \text { and } \widetilde{a} \geq 0\} .
$$


When we refer to an interval $C \in \mathcal{K}_{C}$, its elements are denoted as $c^{-}, c^{+}$, $\widehat{c}, \widetilde{c}$, with $\widetilde{c} \geq 0, c^{-} \leq c^{+}$and the interval by $C=\left[c^{-}, c^{+}\right]$in extreme-point representation and by $C=(\widehat{c} ; \widetilde{c})$, in midpoint notation.

Given $A=\left[a^{-}, a^{+}\right], B=\left[b^{-}, b^{+}\right] \in \mathcal{K}_{C}$ and $\tau \in \mathbb{R}$, we have the following classical (Minkowski-type) addition, scalar multiplication and difference:

- $A+B=\left[a^{-}+b^{-}, a^{+}+b^{+}\right]$,

$-\tau A=\{\tau a: a \in A\}=\left\{\begin{array}{l}{\left[\tau a^{-}, \tau a^{+}\right], \text {if } \tau \geq 0,} \\ {\left[\tau a^{+}, \tau a^{-}\right], \text {if } \tau \leq 0}\end{array}\right.$,

$-{ }_{-} A=(-1) A=\left[-a^{+},-a^{-}\right]$,

- $A-B=A+(-1) B=\left[a^{-}-b^{+}, a^{+}-b^{-}\right]$.

Using midpoint notation, the previous operations, for $A=(\widehat{a} ; \widetilde{a}), B=(\widehat{b} ; \widetilde{b})$ and $\tau \in \mathbb{R}$ are:

$-A+B=(\widehat{a}+\widehat{b} ; \widetilde{a}+\widetilde{b})$,

- $\tau A=(\tau \widehat{a} ;|\tau| \widetilde{a})$,

$-A=(-\widehat{a} ; \widetilde{a})$,

$-A-B=(\widehat{a}-\widehat{b} ; \widetilde{a}+\widetilde{b})$.

We denote the generalized Hukuhara difference ( $g H$-difference in short) of two intervals $A$ and $B$ as $A \ominus_{g H} B=C \Longleftrightarrow(A=B+C$ or $B=A-C)$; the $g H$-difference of two intervals always exists and, in midpoint notation, is equal to

$$
A \ominus_{g H} B=(\widehat{a}-\widehat{b} ;|\widetilde{a}-\widetilde{b}|) \subseteq A-B .
$$

The $g H$-addition for intervals is defined by

$$
A \oplus_{g H} B=A \ominus_{g H}(-B)=(\widehat{a}+\widehat{b} ;|\widetilde{a}-\widetilde{b}|) \subseteq A+B .
$$

If $A \in \mathcal{K}_{C}$, we will denote by $\operatorname{len}(A)=a^{+}-a^{-}=2 \widehat{a}$ the length of interval $A$. Remark that $\alpha A-\beta A=(\alpha+\beta) A$ only if $\alpha \beta \geq 0$ (except for trivial cases) and that $A \ominus_{g H} B=A-B$ or $A \oplus_{g H} B=A+B$ only if $A$ or $B$ are singletons.

For two intervals $A, B \in \mathcal{K}_{C}$ the Pompeiu-Hausdorff distance $d_{H}: \mathcal{K}_{C} \times$ $\mathcal{K}_{C} \rightarrow \mathbb{R}_{+} \cup\{0\}$ is defined by

$$
d_{H}(A, B)=\max \left\{\max _{a \in A} d(a, B), \max _{b \in B} d(b, A)\right\}
$$

with $d(a, B)=\min _{b \in B}|a-b|$. The following properties are well known:

$$
\begin{aligned}
d_{H}(\tau A, \tau B) & =|\tau| d_{H}(A, B), \forall \tau \in \mathbb{R}, \\
d_{H}(A+C, B+C) & =d_{H}(A, B), \text { for all } C \in \mathcal{K}_{C}, \\
d_{H}(A+B, C+D) & \leq d_{H}(A, C)+d_{H}(B, D) .
\end{aligned}
$$

It is known (see $[4,8]$ ) that $d_{H}(A, B)=\left\|A \ominus_{g H} B\right\|$ where for $C \in \mathcal{K}_{C}$, the quantity $\|C\|=\max \{|c| ; c \in C\}=d_{H}(C,\{0\})$ is called the magnitude of $C$; an immediate property of the $g H$-difference for $A, B \in \mathcal{K}_{C}$ is

$$
d_{H}(A, B)=0 \Longleftrightarrow A \ominus_{g H} B=0 \Longleftrightarrow A=B .
$$


It is also well known that $\left(\mathcal{K}_{C}, d_{H}\right)$ is a complete metric space.

A fuzzy set on $\mathbb{R}$ is a mapping $u: \mathbb{R} \rightarrow[0,1]$. we denote its $\alpha$-level set as $[u]_{\alpha}=\{x \in \mathbb{R}: u(x) \geq \alpha\}$ for any $\alpha \in[0,1]$. The $\operatorname{supp}(u)=\{x \in \mathbb{R} \mid u(x)>0\} ;$ 0 -level set of $\mathrm{u}$ is defined by $[u]_{0}=\operatorname{cl}(\operatorname{supp}(u))$ where $\operatorname{cl}(M)$ means the closure of the subset $M \subset \mathbb{R}$.

A fuzzy set $u$ on $\mathbb{R}$ is said to be a fuzzy number if:

(i) $u$ is normal, i.e., there exists $x_{0} \in \mathbb{R}$ such that $u\left(x_{0}\right)=1$,

(ii) $u$ is a convex fuzzy set (i.e. $u(t x+(1-t) y) \geq \min \{u(x), u(y)\}, \forall t \in$ $[0,1], x, y \in \mathbb{R})$,

(iii) $u$ is upper semi-continuous on $\mathbb{R}$,

(iv) $\operatorname{cl}\{x \in \mathbb{R} \mid u(x)>0\}$ is compact.

Let $\mathbb{R}_{F}$ denote the family of fuzzy numbers. So, for any $u \in \mathbb{R}_{F}$ we have $[u]_{\alpha} \in \mathbb{K}_{C}$ for all $\alpha \in[0,1]$ and thus the $\alpha$-levels of a fuzzy number are given by $[u]_{\alpha}=\left[u_{\alpha}^{-}, u_{\alpha}^{+}\right], u_{\alpha}^{-}, u_{\alpha}^{+} \in \mathbb{R}$ for all $\alpha \in[0,1]$. In midpoint notation, we will write $[u]_{\alpha}=\left(\widehat{u}_{\alpha}, \widetilde{u}_{\alpha}\right)$ where $\widehat{u}_{\alpha}=\frac{u_{\alpha}^{+}+u_{\alpha}^{-}}{2}$ and $\widetilde{u}_{\alpha}=\frac{u_{\alpha}^{+}-u_{\alpha}^{-}}{2}$ so that $u_{\alpha}^{-}=\widehat{u}_{\alpha}-\widetilde{u}_{\alpha}$ and $u_{\alpha}^{+}=\widetilde{u}_{\alpha}+\widehat{u}_{\alpha}$. If $[u]_{1}$ is a singleton then we say that $u$ is a fuzzy number. Triangular fuzzy numbers are a special type of fuzzy numbers which are well determined by three real numbers $a \leq b \leq c$, denoted by $u=\langle a, b, c\rangle$, with $\alpha$-levels $[u]_{\alpha}=[a+(b-a) \alpha, c-(c-b) \alpha]$ for all $\alpha \in[0,1]$.

It is well known that in terms of $\alpha$-levels and taking into account the midpoint notation, for every $\alpha \in[0,1][u+v]_{\alpha}=[u]_{\alpha}+[v]_{\alpha}=\left[u_{\alpha}^{-}+\nu_{\alpha}^{-}, u_{\alpha}^{+}+\nu_{\alpha}^{+}\right]=$ $\left(\widehat{u}_{\alpha}+\widehat{\nu}_{\alpha} ; \widetilde{u}_{\alpha}+\widetilde{\nu}_{\alpha}\right)$ and $[\lambda u]_{\alpha}=\left[\min \left\{\lambda u_{\alpha}^{-}, \lambda u_{\alpha}^{+}\right\}, \max \left\{\lambda u_{\alpha}^{-}, \lambda u_{\alpha}^{+}\right\}\right]=\left(\lambda \widehat{u}_{\alpha} ;|\lambda| \widetilde{u}_{\alpha}\right)$.

The following $\mathrm{LgH}$-difference is somewhat more general than the $\mathrm{gH}$ difference:

Definition 1. For given two fuzzy numbers $u$, $\nu$, the level-wise generalized Hukuhara difference (LgH-difference, for short) of $u, \nu$ is defined as the set of interval-valued $g H$-differences

$$
u \ominus_{L g H} \nu=\left\{w_{\alpha} \in \mathcal{K}_{C} \mid w_{\alpha}=[u]_{\alpha} \ominus_{g H}[\nu]_{\alpha} \text { for } \alpha \in[0,1]\right\},
$$

that is, for each $\alpha \in[0,1]$, either $[u]_{\alpha}=[\nu]_{\alpha}+w_{\alpha}$ or $[\nu]_{\alpha}=[u]_{\alpha}-w_{\alpha}$.

\subsection{Orders for Fuzzy Numbers}

The LU-fuzzy partial order is well known in the literature. Let us recall that given $u, \nu \in \mathbb{R}_{\mathcal{F}}$ and given $\alpha \in[0,1]$, heir $\alpha$-levels are $u_{\alpha}=\left[u_{\alpha}^{-}, u_{\alpha}^{+}\right] \in \mathbb{K}_{C}$ and $\nu=\left[\nu_{\alpha}^{-}, \nu_{\alpha}^{+}\right] \in \mathbb{K}_{C}$, respectively.

Definition 2. [7] Given $u, \nu \in \mathbb{R}_{\mathcal{F}}$ and given $\alpha \in[0,1]$, we say that

(i) $u \precsim \alpha-L U \nu$ if and only if $u_{\alpha} \precsim L U \nu_{\alpha}$, that is, $u_{\alpha}^{-} \leq \nu_{\alpha}^{-}$and $u_{\alpha}^{+} \leq \nu_{\alpha}^{+}$,

(ii) $u \preceq_{\alpha-L U} \nu$ if and only if $u_{\alpha} \preceq_{L U} \nu_{\alpha}$,

(iii) $u \prec_{\alpha-L U} \nu$ if and only if $u_{\alpha} \prec_{L U} \nu_{\alpha}$. 
Correspondingly, the analogous LU-fuzzy orders can be obtained by

(a) $u \precsim L U \nu$ if and only if $u \precsim \alpha-L U \nu$ for all $\alpha \in[0,1]$,

(b) $u \preceq_{L U} \nu$ if and only if $u \preceq_{\alpha-L U} \nu$ for all $\alpha \in[0,1]$,

(c) $u \prec_{L U} \nu$ if and only if $u \prec_{\alpha-L U} \nu$ for all $\alpha \in[0,1]$.

The corresponding reverse orders are, respectively, $u \succsim_{L U} \nu \Longleftrightarrow \nu \precsim_{L U} u$, $u \succeq_{L U} \nu \Longleftrightarrow \nu \preceq_{L U} u$ and $u \succ_{L U} \nu \Longleftrightarrow \nu \prec_{L U} u$.

Using $\alpha$-levels midpoint notation $u_{\alpha}=\left(\widehat{u}_{\alpha} ; \widetilde{u}_{\alpha}\right), \nu_{\alpha}=\left(\widehat{\nu}_{\alpha} ; \widetilde{\nu}_{\alpha}\right)$ for all $\alpha \in$ $[0,1]$, the partial orders $(a)$ and $(c)$ above can be expressed for all $\alpha \in[0,1]$ as

$$
\begin{gathered}
\left(L U_{a}\right)\left\{\begin{array}{c}
\widehat{u}_{\alpha} \leq \widehat{\nu}_{\alpha} \\
\widetilde{\nu}_{\alpha} \leq \widetilde{u}_{\alpha}+\left(\widehat{\nu}_{\alpha}-\widehat{u}_{\alpha}\right) \text { and } \\
\widetilde{\nu}_{\alpha} \geq \widetilde{u}_{\alpha}-\left(\widehat{\nu}_{\alpha}-\widehat{u}_{\alpha}\right)
\end{array}\right. \\
\left(L U_{c}\right)\left\{\begin{array}{c}
\widehat{u}_{\alpha}<\widehat{\nu}_{\alpha} \\
\widetilde{\nu}_{\alpha}<\widetilde{u}_{\alpha}+\left(\widehat{\nu}_{\alpha}-\widehat{u}_{\alpha}\right) ; \\
\widetilde{\nu}_{\alpha}>\widetilde{u}_{\alpha}-\left(\widehat{\nu}_{\alpha}-\widehat{u}_{\alpha}\right)
\end{array}\right.
\end{gathered}
$$

the partial order $(b)$ can be expressed in terms of $\left(L U_{a}\right)$ with the additional requirement that at least one of the inequalities is strict.

In the sequel, the results are expressed without proof because they are similar to the ones in [12] and [13]. For the family of intervals $u \ominus_{L g H} \nu$ we write $u \ominus_{L g H} \nu \precsim_{L U} 0$ (and similarly with other orders) to mean that $w_{\alpha} \precsim_{L U} 0$ for all $w_{\alpha} \in u \ominus_{L g H} \nu$.

Proposition 1. Let $u, \nu \in \mathbb{R}_{\mathcal{F}}$ with $u_{\alpha}=\left(\widehat{u}_{\alpha} ; \widetilde{u}_{\alpha}\right), \nu_{\alpha}=\left(\widehat{\nu}_{\alpha} ; \widetilde{\nu}_{\alpha}\right)$ for all $\alpha \in$ $[0,1]$. We have

$$
\begin{aligned}
& \text { (i.a) } u \precsim_{L U} \nu \Leftrightarrow u \ominus_{L g H} \nu \precsim_{L U} 0, \\
& \text { (ii.a) } u \preceq_{L U} \nu \Leftrightarrow u \ominus_{L g H} \nu \preceq_{L U} 0, \\
& \text { (iii.a) } u \prec_{L U} \nu \Leftrightarrow u \ominus_{L g H} \nu \prec_{L U} 0, \\
& \text { (iv.a) } u \prec_{L U} \nu \Longrightarrow u \preceq_{L U} \nu \Longrightarrow u \precsim_{L U} \nu, \\
& \text { (i.b) } u \succsim_{L U} \nu \Leftrightarrow u \ominus_{L g H} \nu \succsim_{L U} 0, \\
& \text { (ii.b) } u \succeq_{L U} \nu \Leftrightarrow u \ominus_{L g H} \nu \succeq_{L U} 0, \\
& \text { (iii.b) } u \succ_{L U} \nu \Leftrightarrow u \ominus_{L g H} \nu \succ_{L U} 0, \\
& \text { (iv.b) } u \succ_{L U} \nu \Longrightarrow u \succeq_{L U} \nu \Longrightarrow u \succsim_{L U} \nu .
\end{aligned}
$$

We say that $u$ and $\nu$ are LU-incomparable if neither $u \precsim_{L U} \nu$ nor $u \succsim_{L U} \nu$ and $u$ and $\nu$ are $\alpha$-LU-incomparable if neither $u \precsim_{\alpha-L U} \nu$ nor $u \succsim_{\alpha-L U} \nu$.

Proposition 2. Let $u, \nu \in \mathbb{R}_{\mathcal{F}}$ with $u_{\alpha}=\left(\widehat{u}_{\alpha} ; \widetilde{u}_{\alpha}\right), \nu_{\alpha}=\left(\widehat{\nu}_{\alpha} ; \widetilde{\nu}_{\alpha}\right)$ for all $\alpha \in$ $[0,1]$. The following are equivalent:

(i) $u$ and $\nu$ are $\alpha$-LU-incomparable;

(ii) $u_{\alpha} \ominus_{g H} \nu_{\alpha}$ is not a singleton and $0 \in \operatorname{int}\left(u_{\alpha} \ominus_{g H} \nu_{\alpha}\right)$;

(iii) $\left|\widehat{u}_{\alpha}-\widehat{\nu}_{\alpha}\right|<\left|\widetilde{\nu}_{\alpha}-\widetilde{u}_{\alpha}\right|$ for $\alpha \in[0,1]$;

(iv) $u_{\alpha} \subset \operatorname{int}\left(\nu_{\alpha}\right)$ or $\nu_{\alpha} \subset \operatorname{int}\left(u_{\alpha}\right)$. 
Proposition 3. If $u, \nu, w \in \mathbb{R}_{\mathcal{F}}$, then

(i) $u \precsim L U \nu$ if and only if $(u+w) \precsim L U(v+w)$;

(ii-a) If $u+\nu \precsim_{L U} w$ then $u \precsim_{L U}\left(w \ominus_{L g H} \nu\right)$;

(ii-b) If $u+\nu \succsim_{L U} w$ then $u \succsim_{L U}\left(w \ominus_{L g H} \nu\right)$;

(iii) $u \precsim_{L U} \nu$ if and only if $(-\nu) \precsim_{L U}(-u)$.

\section{Fuzzy-Valued Functions}

A function $F:[a, b] \longrightarrow \mathbb{R}_{F}$ is said to be a fuzzy-valued function. For any $\alpha \in[0,1]$, associated to $F$, we define the family of interval-valued functions $F_{\alpha}:[a, b] \longrightarrow \mathbb{K}_{C}$ given by $[F(x)]_{\alpha}=\left[f_{\alpha}^{-}(x), f_{\alpha}^{+}(x)\right]$ for all $\alpha \in[0,1]$. In midpoint representation, we write $[F(x)]_{\alpha}=\left(\widehat{f}_{\alpha}(x) ; \widetilde{f}_{\alpha}(x)\right)$ where $\widehat{f}_{\alpha}(x) \in \mathbb{R}$ is the midpoint value of interval $[F(x)]_{\alpha}$ and $\widetilde{f}_{\alpha}(x) \in \mathbb{R}^{+} \cup\{0\}$ is the nonnegative half-length of $F_{\alpha}(x)$ :

$$
\begin{aligned}
& \widehat{f}_{\alpha}(x)=\frac{f_{\alpha}^{+}(x)+f_{\alpha}^{-}(x)}{2} \text { and } \\
& \widetilde{f}_{\alpha}(x)=\frac{f_{\alpha}^{+}(x)-f_{\alpha}^{-}(x)}{2} \geq 0
\end{aligned}
$$

so that

$$
f_{\alpha}^{-}(x)=\widehat{f}_{\alpha}(x)-\widetilde{f_{\alpha}}(x) \text { and } f_{\alpha}^{+}(x)=\widehat{f}_{\alpha}(x)+\widetilde{f}_{\alpha}(x) .
$$

Proposition 4. Let $F: T \longrightarrow \mathbb{R}_{F}$ be a fuzzy-valued function and $x_{0} \in T \subseteq \mathbb{R}$ be an accumulation point of $T$. If $\lim _{x \rightarrow x_{0}} F(x)=L$ with $L_{\alpha}=\left[l_{\alpha}^{-}, l_{\alpha}^{+}\right]$. Then $\lim _{x \rightarrow x_{0}}[F(x)]_{\alpha}=\left[l_{\alpha}^{-}, l_{\alpha}^{+}\right]$for all $\alpha$ (uniformly in $\alpha \in[0,1]$ ).

In midpoint notation, let $[F(x)]_{\alpha}=\left(\widehat{f}_{\alpha}(x) ; \widetilde{f}_{\alpha}(x)\right)$ and $L_{\alpha}=\left(\widehat{l}_{\alpha} ; \widetilde{l}_{\alpha}\right)$ for all $\alpha \in[0,1]$; then the limits and continuity can be expressed, respectively, as

$$
\lim _{x \rightarrow x_{0}}[F(x)]_{\alpha}=L_{\alpha} \Longleftrightarrow\left\{\begin{array}{c}
\lim _{x \rightarrow x_{0}} \widehat{f}_{\alpha}(x)=\widehat{l}_{\alpha} \\
\lim _{x \rightarrow x_{0}} \tilde{f}_{\alpha}(x)=\tilde{l}_{\alpha}
\end{array}\right.
$$

and

$$
\lim _{x \rightarrow x_{0}}[F(x)]_{\alpha}=\left[F\left(x_{0}\right)\right]_{\alpha} \Longleftrightarrow\left\{\begin{array}{l}
\lim _{x \rightarrow x_{0}} \widehat{f_{\alpha}}(x)=\widehat{f_{\alpha}}\left(x_{0}\right) \\
\lim _{x \rightarrow x_{0}} \widetilde{f_{\alpha}}(x)=\widetilde{f_{\alpha}}\left(x_{0}\right) .
\end{array}\right.
$$

The following proposition connects limits to the order of fuzzy numbers. Analogous results can be obtained for the reverse partial order $\succsim_{L U}$.

Proposition 5. Let $F, G, H: T \longrightarrow \mathbb{R}_{F}$ be fuzzy-valued functions and $x_{0}$ an accumulation point for $T$. 
(i) If $F(x) \precsim_{L U} G(x)$ for all $x \in T$ in a neighborhood of $x_{0}$ and $\lim _{x \rightarrow x_{0}} F(x)=$ $L \in \mathbb{R}_{F}, \lim _{x \rightarrow x_{0}} G(x)=M \in \mathbb{R}_{F}$, then $L \precsim L U M$;

(ii) If $F(x) \precsim_{L U} G(x) \precsim_{L U} H(x)$ for all $x \in T$ in a neighborhood of $x_{0}$ and $\lim _{x \rightarrow x_{0}} F(x)=\lim _{x \rightarrow x_{0}} H(x)=L \in \mathbb{R}_{F}$, then $\lim _{x \rightarrow x_{0}} G(x)=L$.

Similar results as in Propositions 4 and 5 are valid for the left limit with $x \longrightarrow x_{0}, x<x_{0}$ ( $x \nearrow x_{0}$ for short) and for the right limit $x \longrightarrow x_{0}, x>x_{0}$ $\left(x \searrow x_{0}\right.$ for short); the condition that $\lim _{x \rightarrow x_{0}} F(x)=L$ if and only if $\lim _{x \nearrow x_{0}} F(x)=$ $L=\lim _{x \backslash x_{0}} F(x)$ is obvious.

The graphical representation of a fuzzy-valued function is then possible in terms either of the standard way, by picturing the level curves $y=f_{\alpha}^{-}(x)$ and $y=f_{\alpha}^{+}(x)$ in the plane $(x, y)$, or, in the half-plane $(\widehat{z} ; \widetilde{z})$, by plotting the parametric curves $\widehat{z}=\widehat{f}_{\alpha}(x)$ and $\widetilde{z}=\widetilde{f}_{\alpha}(x)$; Figs. 1 and 2 give an illustration of the two graphical alternatives for the (periodic, with period $2 \pi$ ) fuzzy function $F(x)$ having $\alpha$-cuts defined by functions $\widehat{f}_{\alpha}(x)=5 \cos (x)-(\sqrt{2}-1) \cos (5 x)$ and $\widetilde{f}_{\alpha}(x)=(1-0.5 \alpha)^{2}(1.5+\sin (4 x))$ for $x \in[0,2 \pi]$; only $n=11 \alpha$-cuts are pictured for uniform $\alpha \in\left\{\frac{i-1}{10} \mid i=1,2, \ldots, n\right\}$ (see also Fig. 3).

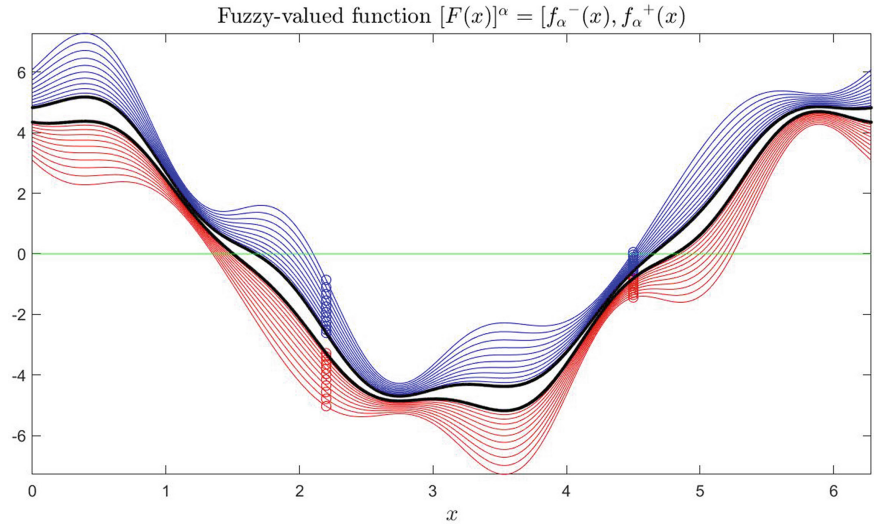

Fig. 1. Level-wise endpoint graphical representation of the fuzzy-valued function with $\alpha$-cuts $[F(x)]_{\alpha}=\left[\widehat{f}_{\alpha}(x)-\widetilde{f}_{\alpha}(x), \widehat{f}_{\alpha}(x)+\widetilde{f}_{\alpha}(x)\right]$ where $\widehat{f}_{\alpha}(x)=5 \cos (x)-(\sqrt{2}-$ $1) \cos (5 x)$ and $\tilde{f}_{\alpha}(x)=(1-0.6 \alpha)^{2}(1.5+\sin (4 x))$ for $x \in[0,2 \pi]$. The core, intercepted by the black-colored curves, is the interval-valued function $\left.x \longrightarrow[F(x)]_{1}\right]=$ $\left[f_{1}^{-}(x), f_{1}^{+}(x)\right]$. The other $\alpha$-cuts are represented by red-colored curves for the left extreme functions $f_{\alpha}^{-}(x)$ and blue-colored curves for the right extreme functions $f_{\alpha}^{+}(x)$. The marked points correspond to $x=2.2$ and $x=4.5$. (Color figure online)

In relation with the $\mathrm{LgH}$-difference, we consider the concept of $\mathrm{LgH}$ differentiability. 


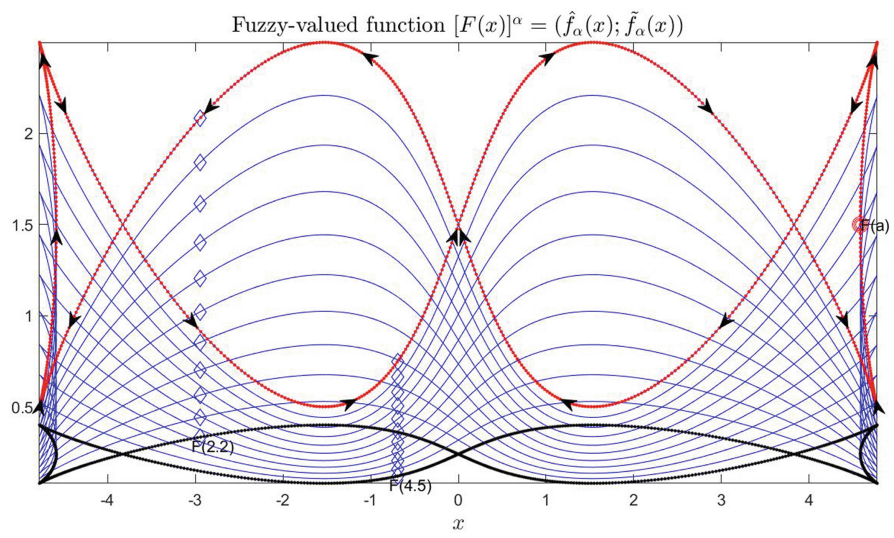

Fig. 2. Level-wise midpoint graphical representation in the half plane $(\widehat{z} ; \widetilde{z})$ with $\widetilde{z} \geq 0$ as vertical axis, of the fuzzy-valued function $F(x), x \in[0,2 \pi]$, described in Fig. 1 . In this representation, each curve corresponds to a single $\alpha$-cut (only $n=11$ curves are pictured with uniform $\alpha=0,0.1, \ldots, 1$ ); the core corresponds to the black-colored curve, the support to the red-colored one. The arrows give the direction of $x$ from initial 0 to final $2 \pi$. The marked points correspond to $x=2.2$ and $x=4.5$. (Color figure online)
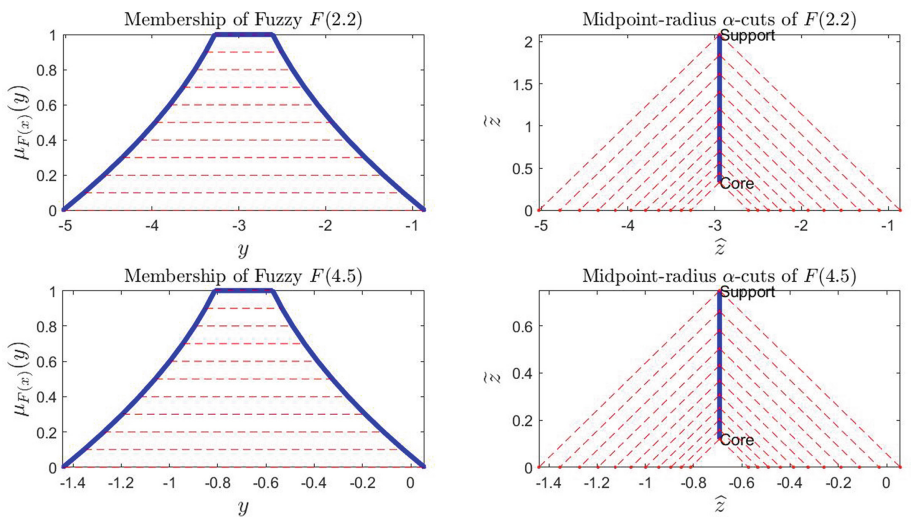

Fig. 3. Membership function and level-wise midpoint representations of two values $F(2.2)$ and $F(4.5)$ of the fuzzy-valued function $F(x)$ described in Fig. 1. In the midpoint representation, a vertical curve corresponds to the displacement of the $n=11$ computed $\alpha$-cuts; the red lines on the right pictures reconstruct the $\alpha$-cuts. Remark that $y$ and $\widehat{z}$ represent the same domain and that a linear vertical segment in the midpoint representation corresponds to a symmetric membership function having the same value of $\widehat{f}_{\alpha}(x)$ for all $\alpha$. (Color figure online)

Definition 3. [6] Let $\left.x_{0} \in\right] a, b\left[\right.$ and $h$ be such that $\left.x_{0}+h \in\right] a, b[$, then the levelwise $g H$-derivative (LgH-differentiable for short) of a function $F:] a, b\left[\rightarrow \mathbb{R}_{F}\right.$ at 
$x_{0}$ is defined as the set of interval-valued $g H$-derivatives, if they exist,

$$
F_{L g H}^{\prime}\left(x_{0}\right)_{\alpha}=\lim _{h \rightarrow 0} \frac{1}{h}\left(\left[F\left(x_{0}+h\right)\right]_{\alpha} \ominus_{g H}\left[F\left(x_{0}\right)\right]_{\alpha}\right)
$$

if $F_{L g H}^{\prime}\left(x_{0}\right)_{\alpha}$ is a compact interval for all $\alpha \in[0,1]$, we say that $F$ is levelwise generalized Hukuhara differentiable (LgH-differentiable for short) at $x_{0}$ and the family of intervals $\left\{F_{L g H}^{\prime}\left(x_{0}\right)_{\alpha} \mid \alpha \in[0,1]\right\}$ is the LgH-derivative of $F$ at $x_{0}$, denoted by $F_{L g H}^{\prime}\left(x_{0}\right)$.

Also, one-side derivatives can be considered. The right $\mathrm{LgH}$-derivative of $F$ at $x_{0}$ is $F_{(r) g H}^{\prime}\left(x_{0}\right)_{\alpha}=\lim _{h \searrow 0} \frac{1}{h}\left(\left[F\left(x_{0}+h\right)\right]_{\alpha} \ominus_{g H}\left[F\left(x_{0}\right)\right]_{\alpha}\right)$ while to the left it is defined as $F_{(l) L g H}^{\prime}\left(x_{0}\right)_{\alpha}=\lim _{h \nearrow 0} \frac{1}{h}\left(\left[F\left(x_{0}+h\right)\right]_{\alpha} \ominus_{g H}\left[F\left(x_{0}\right)\right]_{\alpha}\right)$. The LgHderivative exists at $x_{0}$ if and only if the left and right derivatives at $x_{0}$ exist and are the same interval.

In terms of midpoint representation $[F(x)]_{\alpha}=\left(\widehat{f_{\alpha}}(x) ; \widetilde{f_{\alpha}}(x)\right)$, for all $\alpha \in$ $[0,1]$, we can write

$$
\begin{aligned}
\frac{[F(x+h)]_{\alpha} \ominus_{g H}[F(x)]_{\alpha}}{h} & =\left(\widehat{\Delta}_{g H} F_{\alpha}(x, h) ; \widetilde{\Delta}_{g H} F_{\alpha}(x, h)\right), \text { where } \\
\widehat{\Delta}_{g H} F_{\alpha}(x, h) & =\frac{\widehat{f_{\alpha}}(x+h)-\widehat{f_{\alpha}}(x)}{h} \\
\widetilde{\Delta}_{g H} F_{\alpha}(x, h) & =\left|\frac{\widetilde{f_{\alpha}}(x+h)-\widetilde{f_{\alpha}}(x)}{h}\right|
\end{aligned}
$$

and taking the limit for $h \longrightarrow 0$, we obtain the $\mathrm{LgH}$-derivative of $F_{\alpha}$, if and only if the two limits $\lim _{h \longrightarrow 0} \frac{\widehat{f_{\alpha}}(x+h)-\widehat{f_{\alpha}}(x)}{h}$ and $\lim _{h \longrightarrow 0}\left|\frac{\widetilde{f_{\alpha}}(x+h)-\widetilde{f_{\alpha}}(x)}{h}\right|$ exist in $\mathbb{R}$; remark that the midpoint function $\widehat{f_{\alpha}}$ is required to admit the ordinary derivative at $x$. With respect to the existence of the second limit, the existence of the left and right derivatives $\tilde{f}_{(l) \alpha}^{\prime}(x)$ and $\tilde{f}_{(r) \alpha}^{\prime}(x)$ is required with $\left|\tilde{f}_{(l) \alpha}^{\prime}(x)\right|=\left|\tilde{f}_{(r) \alpha}^{\prime}(x)\right|=$ $\widetilde{w}_{\alpha}(x) \geq 0$ (in particular $\widetilde{w}_{\alpha}(x)=\left|{\widetilde{f_{\alpha}}}^{\prime}(x)\right|$ if ${\widetilde{f_{\alpha}}}^{\prime}(x)$ exists) so that we have

$$
F_{L g H}^{\prime}(x)_{\alpha}=\left({\widehat{f_{\alpha}}}^{\prime}(x) ; \widetilde{w}_{\alpha}(x)\right)
$$

or, in the standard interval notation,

$$
F_{L g H}^{\prime}(x)_{\alpha}=\left[{\widehat{f_{\alpha}}}^{\prime}(x)-\widetilde{w}_{\alpha}(x),{\widehat{f_{\alpha}}}^{\prime}(x)+\widetilde{w}_{\alpha}(x)\right] .
$$

\section{Monotonicity of Functions with Values in $\left(\mathbb{R}_{F}, L U\right)$}

Monotonicity of fuzzy-valued functions has not been much investigated and this is partially due to the lack of unique meaningful definition of an order for fuzzyvalued functions. We can analyze monotonicity and, using the gH-difference, related characteristics of inequalities for fuzzy-valued functions. 
Definition 4. Let $F:[a, b] \rightarrow \mathbb{R}_{F}$ be fuzzy function, where $[F(x)]_{\alpha}=$ $\left(\widehat{f_{\alpha}}(x) ; \widetilde{f_{\alpha}}(x)\right)$ for all $\alpha \in[0,1]$. We say that $F$ is

(a-i) (るLU)-nondecreasing on $[a, b]$ if $x_{1}<x_{2}$ implies $F\left(x_{1}\right) \precsim_{L U} F\left(x_{2}\right)$ for all $x_{1}, x_{2} \in[a, b]$;

(a-ii) (るLU)-nonincreasing on $[a, b]$ if $x_{1}<x_{2}$ implies $F\left(x_{2}\right) \precsim_{L U} F\left(x_{1}\right)$ for all $x_{1}, x_{2} \in[a, b]$;

(b-i) (strictly) $\left(\preceq_{L U}\right)$-increasing on $[a, b]$ if $x_{1}<x_{2}$ implies $F\left(x_{1}\right) \preceq_{L U} F\left(x_{2}\right)$ for all $x_{1}, x_{2} \in[a, b]$;

(b-ii) (strictly) ( $\left.\swarrow_{L U}\right)$-decreasing on $[a, b]$ if $x_{1}<x_{2}$ implies $F\left(x_{2}\right) \preceq_{L U} F\left(x_{1}\right)$ for all $x_{1}, x_{2} \in[a, b]$;

(c-i) (strongly) $\left(\prec_{L U}\right)$-increasing on $[a, b]$ if $x_{1}<x_{2}$ implies $F\left(x_{1}\right) \prec_{L U} F\left(x_{2}\right)$ for all $x_{1}, x_{2} \in[a, b]$;

(c-ii) (strongly) $\left(\prec_{L U}\right)$-decreasing on $[a, b]$ if $x_{1}<x_{2}$ implies $F\left(x_{2}\right) \prec_{L U} F\left(x_{1}\right)$ for all $x_{1}, x_{2} \in[a, b]$.

If one of the six conditions is satisfied, we say that $F$ is monotonic on $[a, b]$; the monotonicity is strict if (b-i,b-ii) or strong if (c-i,c-ii) are satisfied.

The monotonicity of $F:[a, b] \rightarrow \mathbb{R}_{F}$ can be analyzed also locally, in a neighborhood of an internal point $\left.x_{0} \in\right] a, b\left[\right.$, by considering condition $F(x) \precsim_{L U} F\left(x_{0}\right)$ (or condition $F(x) \succsim_{L U} F\left(x_{0}\right)$ ) for $\left.x \in\right] a, b\left[\right.$ and $\left|x-x_{0}\right|<\delta$ with a positive small $\delta$. We omit the corresponding definitions as they are analogous to the previous ones.

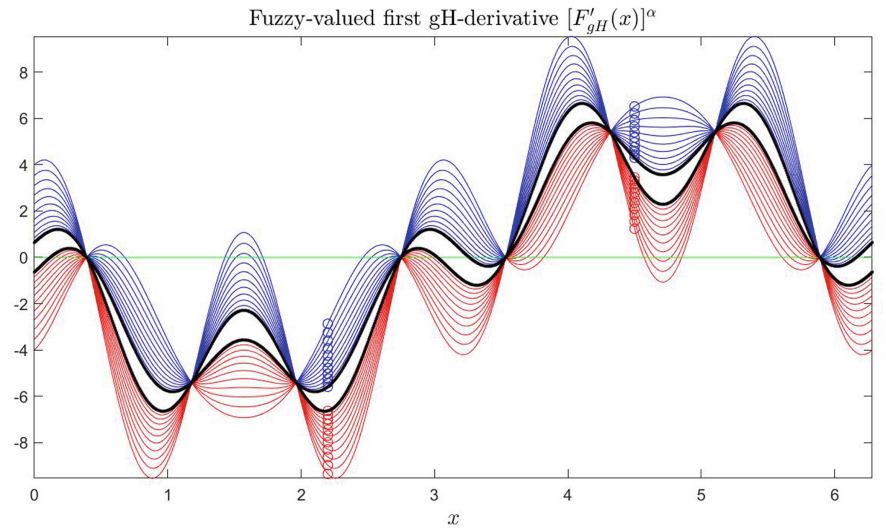

Fig. 4. Level-wise endpoint graphical representation of the fuzzy-valued LgH-derivative $\left.\left[F_{L g H}^{\prime}\right](x)\right]_{\alpha}=\left[\widehat{f}_{\alpha}^{\prime}(x)-\left|\widetilde{f}_{\alpha}^{\prime}(x)\right|, \widehat{f}_{\alpha}^{\prime}(x)+\left|\widetilde{f}_{\alpha}^{\prime}(x)\right|\right]$ where $\widehat{f}_{\alpha}(x)=5 \cos (x)-(\sqrt{2}-$ 1) $\cos (5 x)$ and $\widetilde{f}_{\alpha}(x)=(1-0.6 \alpha)^{2}(1.5+\sin (4 x))$ for $x \in[0,2 \pi]$. The core is intercepted by the black-colored curves. The other $\alpha$-cuts are represented by red-colored curves for the left extreme functions and blue-colored curves for the right extreme functions. The marked points correspond to $x=2.2$ and $x=4.5$. (Color figure online) 
Proposition 6. Let $F:[a, b] \rightarrow \mathbb{R}_{F}$ be fuzzy function, where $F_{\alpha}(x)=\left(\widehat{f_{\alpha}}(x)\right.$; $\left.\widetilde{f_{\alpha}}(x)\right)$ for $\alpha \in[0,1]$ and $\left.x_{0} \in\right] a, b[$. Then

(i) $F(x)$ is $(\precsim L U)$-nondecreasing at $x_{0}$ if and only if $\widehat{f_{\alpha}}(x)$ is nondecreasing, $\widetilde{f_{\alpha}}(x)-\widehat{f_{\alpha}}(x)$ is nonincreasing and $\widetilde{f_{\alpha}}(x)+\widehat{f_{\alpha}}(x)$ is nondecreasing at $x_{0}$ for all $\alpha \in[0,1]$;

(ii) $F(x)$ is $\left(\precsim^{\precsim} L U\right)$-nonincreasing at $x_{0}$ if and only if $\widehat{f_{\alpha}}(x)$ is nonincreasing, $\widetilde{f_{\alpha}}(x)-\widehat{f_{\alpha}}(x)$ is nondecreasing and $\widetilde{f_{\alpha}}(x)+\widehat{f_{\alpha}}(x)$ is nonincreasing at $x_{0}$ for all $\alpha \in[0,1]$;

(iii) Analogous conditions are valid for strict and strong monotonicity.

Proposition 7. Let $F:] a, b\left[\rightarrow \mathbb{R}_{F}\right.$ be fuzzy function, where $[F(x)]_{\alpha}=$ $\left(\widehat{f_{\alpha}}(x) ; \widetilde{f_{\alpha}}(x)\right)$ for all $\alpha \in[0,1]$ and let $F$ be LgH-differentiable at the internal points $x \in] a, b[$. Then

(1) If $F$ is $\left(\precsim_{L U}\right)$-nondecreasing on $] a, b\left[\right.$, then for all $x, F_{L g H}^{\prime}(x) \succsim_{L U} 0$;

(2) If $F$ is $\left(\precsim_{L U}\right)$-nonincreasing on $] a, b\left[\right.$, then for all $x, F_{L g H}^{\prime}(x) \precsim_{L U} 0$.

In Fig. 4 we picture the fuzzy-valued first order $\mathrm{LgH}$-derivative of a function $F(x)$ and in Fig. 5 we show graphically the membership functions of $F_{L g H}^{\prime}(x)$ at two points $x=2.2$ and $x=4.5$. Observe from Fig. 5 that $F_{L g H}^{\prime}(4.5)$ is positive in the $(\precsim L U)$ order and that $F_{L g H}^{\prime}(2.2)$ is negative in the same order relation, denoting that $F(x)$ is locally strictly increasing around $x=4.5$ and locally strictly decreasing around $x=2.2$ (see also Fig. 4 ).
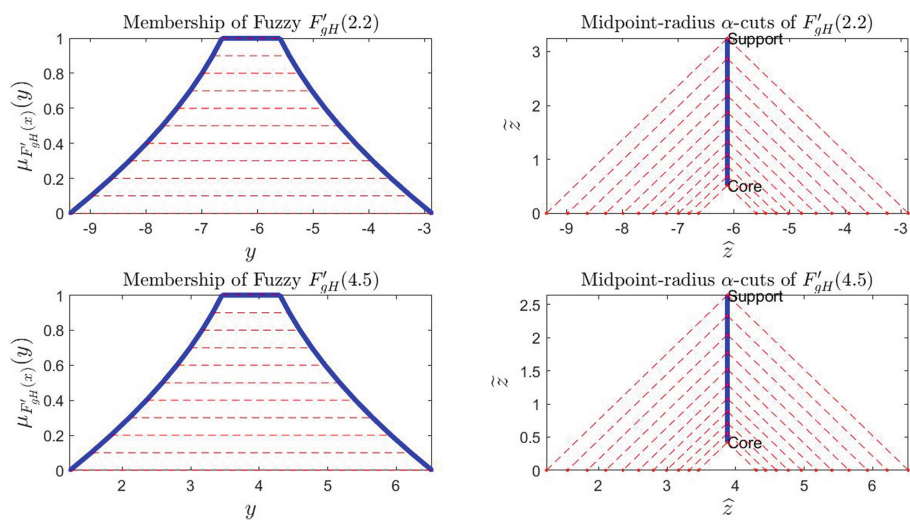

Fig. 5. Membership function and level-wise midpoint representations of two values $F_{L g H}^{\prime}(2.2)$ and $F_{L g H}^{\prime}(4.5)$ of the fuzzy-valued $\mathrm{LgH}$-derivative of function $F(x)$ described in Fig. 1. In the midpoint representation, a vertical curve corresponds to the displacement of the $n=11$ computed $\alpha$-cuts; the red lines on the right pictures reconstruct the $\alpha$-cuts. Remark that $y$ and $\widehat{z}$ represent the same domain and that a linear vertical segment in the midpoint representation corresponds to a symmetric membership function. (Color figure online) 
Analogous results are also immediate, relating strong (local) monotonicity of $F$ to the "sign" of its left and right derivatives $F_{(l) L g H}^{\prime}(x)$ and $F_{(r) L g H}^{\prime}(x)$; at the extreme points of $[a, b]$, we consider only right (at $a$ ) or left (at $b$ ) monotonicity and right or left derivatives.

Proposition 8. Let $F:[a, b] \rightarrow \mathbb{R}_{F}$ be fuzzy function, where $[F(x)]_{\alpha}=$ $\left(\widehat{f_{\alpha}}(x) ; \widetilde{f_{\alpha}}(x)\right)$ for all $\alpha \in[0,1]$ with left and/or right $g H$-derivatives at a point $x_{0} \in[a, b]$. Then

(i.a) if $0 \prec_{L U} F_{(l) L g H}^{\prime}\left(x_{0}\right)$, then $F$ is strongly $\left(\prec_{L U}\right)$-increasing on $\left[x_{0}-\delta, x_{0}\right]$ for some $\delta>0$ (here $\left.x_{0}>a\right)$;

(i.b) if $0 \prec_{L U} F_{(r) L g H}^{\prime}\left(x_{0}\right)$, then $F$ is strongly $\left(\prec_{L U}\right)$-increasing on $\left[x_{0}, x_{0}+\delta\right]$ for some $\delta>0$ (here $\left.x_{0}<b\right)$;

(ii.a) if $0 \succ_{L U} F_{(l) L g H}^{\prime}\left(x_{0}\right)$, then $F$ is strongly $\left(\prec_{L U}\right)$-decreasing on $\left[x_{0}-\delta, x_{0}\right]$ for some $\delta>0$ (here $\left.x_{0}>a\right)$;

(ii.b) if $0 \succ_{L U} F_{(r) L g H}^{\prime}\left(x_{0}\right)$, then $F$ is strongly $\left(\prec_{L U}\right)$-decreasing on $\left[x_{0}, x_{0}+\delta\right]$ for some $\delta>0$ (here $\left.x_{0}<b\right)$.

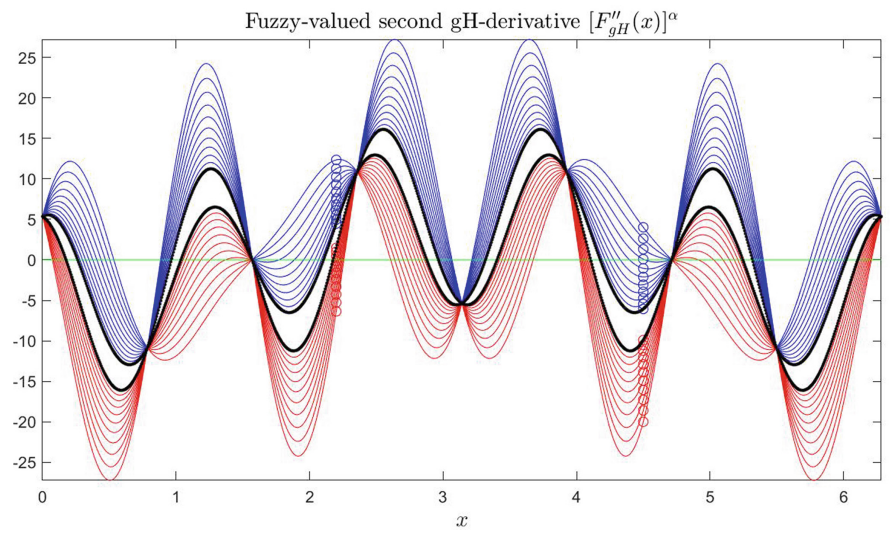

Fig. 6. Level-wise endpoint graphical representation of the fuzzy-valued LgH-derivative $\left.\left[F_{L g H}^{\prime \prime}\right](x)\right]_{\alpha}=\left[\widehat{f}_{\alpha}^{\prime \prime}(x)-\left|\widetilde{f}_{\alpha}^{\prime \prime}(x)\right|, \widehat{f}_{\alpha}^{\prime \prime}(x)+\left|\widetilde{f}_{\alpha}^{\prime \prime}(x)\right|\right]$ where $\widehat{f}_{\alpha}(x)=5 \cos (x)-(\sqrt{2}-$ $1) \cos (5 x)$ and $\tilde{f}_{\alpha}(x)=(1-0.6 \alpha)^{2}(1.5+\sin (4 x))$ for $x \in[0,2 \pi]$. The core is intercepted by the black-colored curves. The other $\alpha$-cuts are represented by red-colored curves for the left extreme functions $f_{\alpha}^{-}(x)$ and blue-colored curves for the right extreme functions $f_{\alpha}^{+}(x)$. (Color figure online)

\section{Concavity and Convexity of Fuzzy-Valued Functions}

We have three types of convexity, similar to the monotonicity and local extremum concepts. 
Definition 5. Let $F:[a, b] \rightarrow \mathbb{R}_{F}$ be a function and let $\precsim L U$ be a partial order on $\mathbb{R}_{F}$. We say that

(a-i) $\quad F$ is $(\precsim L U)$-convex on $[a, b]$ if and only if $F\left((1-\lambda) x_{1}+\lambda x_{2}\right) \precsim L U$ $(1-\lambda) F\left(x_{1}\right)+\lambda F\left(x_{2}\right), \forall x_{1}, x_{2} \in[a, b]$ and all $\lambda \in[0,1]$;

(a-ii) $\quad F$ is $\left(\precsim_{\gamma^{-}, \gamma^{+}}\right)$-concave on $[a, b]$ if and only if $F\left((1-\lambda) x_{1}+\lambda x_{2}\right) \succsim_{L U}$ $(1-\lambda) F\left(x_{1}\right)+\lambda F\left(x_{2}\right), \forall x_{1}, x_{2} \in[a, b]$ and all $\lambda \in[0,1]$.

(b-i) $\quad F$ is strictly $\left(\preceq_{L U}\right)$-convex on $[a, b]$ if and only if $F\left((1-\lambda) x_{1}+\right.$ $\left.\lambda x_{2}\right) \preceq_{L U}(1-\lambda) F\left(x_{1}\right)+\lambda F\left(x_{2}\right), \forall x_{1}, x_{2} \in[a, b]$ and all $\left.\lambda \in\right] 0,1[$;

(b-ii) $\quad F$ is strictly $\left(\preceq_{L U}\right)$-concave on $[a, b]$ if and only if $F\left((1-\lambda) x_{1}+\right.$ $\left.\lambda x_{2}\right) \succsim_{L U}(1-\lambda) F\left(x_{1}\right)+\lambda F\left(x_{2}\right), \forall x_{1}, x_{2} \in[a, b]$ and all $\left.\lambda \in\right] 0,1[$.

(c-i) $\quad F$ is strongly $\left(\prec_{L U}\right)$-convex on $[a, b]$ if and only if $F\left((1-\lambda) x_{1}+\right.$ $\left.\lambda x_{2}\right) \prec_{L U}(1-\lambda) F\left(x_{1}\right)+\lambda F\left(x_{2}\right), \forall x_{1}, x_{2} \in[a, b]$ and all $\left.\lambda \in\right] 0,1[$;

(c-ii) $\quad F$ is strongly $\left(\prec_{L U}\right)$-concave on $[a, b]$ if and only if $F\left((1-\lambda) x_{1}+\right.$ $\left.\lambda x_{2}\right) \succ_{L U}(1-\lambda) F\left(x_{1}\right)+\lambda F\left(x_{2}\right), \forall x_{1}, x_{2} \in[a, b]$ and all $\left.\lambda \in\right] 0,1[$.

The convexity of a function $[F]_{\alpha}=\left(\widehat{f_{\alpha}} ; \widetilde{f_{\alpha}}\right)$ is related to the concavity of function $[-F]_{\alpha}=\left(-\widehat{f_{\alpha}} ; \widetilde{f_{\alpha}}\right)$ for all $\alpha \in[0,1]$. It is easy to see that $F$ is $(\precsim L U)$ convex if and only if $-F$ is $(\precsim L U)$-concave.

Proposition 9. Let $F:[a, b] \rightarrow \mathbb{R}_{F}$ with $[F]_{\alpha}=\left(\widehat{f_{\alpha}} ; \widetilde{f_{\alpha}}\right)$ for all $\alpha \in[0,1]$ and $\precsim_{L U}$ be a given partial order; then

1. F is $(\precsim L U)$-convex if and only if $\widehat{f_{\alpha}}$ is convex, $\widetilde{f_{\alpha}}-\widehat{f_{\alpha}}$ is concave and $\widetilde{f_{\alpha}}+\widehat{f_{\alpha}}$ is convex;

2. F is (るLU)-concave if and only if $\widehat{f_{\alpha}}$ is concave, $\widetilde{f_{\alpha}}-\widehat{f_{\alpha}}$ is convex and $\widetilde{f_{\alpha}}+\widehat{f_{\alpha}}$ is concave.

From Proposition 9 , it is easy to see that $F$ is ( $\precsim L U$ )-convex (or concave) if and only if $f_{\alpha}^{-}$and $f_{\alpha}^{+}$are convex (or concave) for all $\alpha \in[0,1]$. Also, several ways to analyze ( $L U$ )-convexity (or concavity) in terms of the first or second derivatives of functions $\widehat{f_{\alpha}}, \widehat{f_{\alpha}}-\widetilde{f_{\alpha}}$ and $\widetilde{f_{\alpha}}+\widehat{f_{\alpha}}$ can be easily deduced.

Proposition 10. Let $F:] a, b\left[\rightarrow \mathbb{R}_{F}\right.$ with $[F]_{\alpha}=\left(\widehat{f_{\alpha}} ; \widetilde{f_{\alpha}}\right)$ for all $\alpha \in[0,1]$. Real-valued functions $\widehat{f}_{\alpha}$ and $\widetilde{f_{\alpha}}$ are differentiable, then;

1. If the first order derivatives $\widehat{f}_{\alpha}^{\prime}$ and $\widetilde{f}_{\alpha}^{\prime}$ exist, then:

(1-a) $F$ is (るLU)-convex on $] a, b\left[\right.$ if and only if $\widehat{f}_{\alpha}^{\prime}, \widehat{f}_{\alpha}^{\prime}-\widetilde{f}_{\alpha}^{\prime}$ and $\widetilde{f}_{\alpha}^{\prime}+\widehat{f}_{\alpha}^{\prime}$ are increasing (nondecreasing) for all $\alpha \in[0,1]$ on $] a, b[$; 

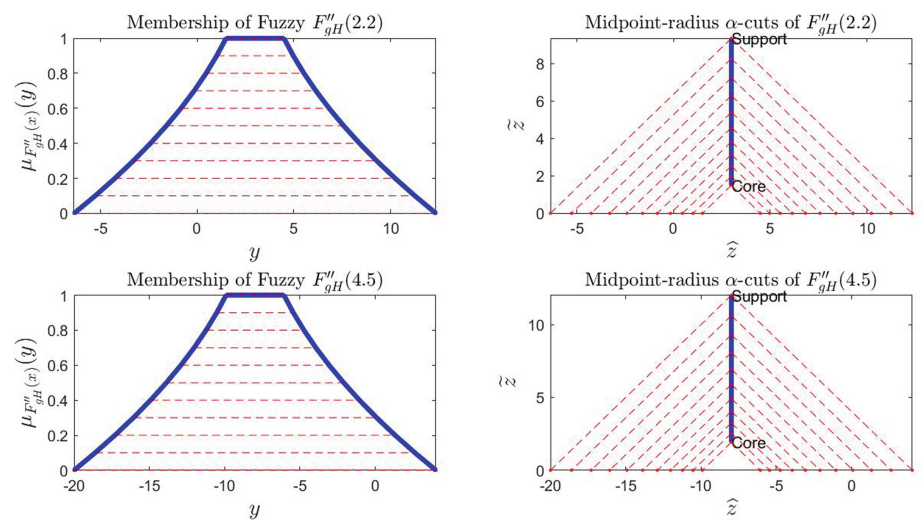

Fig. 7. Membership function and level-wise midpoint representations of two values $F_{L g H}^{\prime \prime}(2.1)$ and $F_{L g H}^{\prime \prime}(4.6)$ of the fuzzy-valued function $F(x)$ described in Fig. 1 . In this midpoint representation, a vertical curve corresponds to the displacement of the $n=11$ computed $\alpha$-cuts; the red lines on the right pictures reconstruct the $\alpha$-cuts. Remark that $y$ and $\widehat{z}$ represent the same domain and that a linear vertical segment in the midpoint representation corresponds to a symmetric membership function, having the same value for all $\alpha \in[0,1]$. (Color figure online)

(1-b) $F$ is (るLU)-concave on $] a, b\left[\right.$ if and only if $\widehat{f}_{\alpha}^{\prime}, \widehat{f}_{\alpha}^{\prime}-\widetilde{f}_{\alpha}^{\prime}$ and $\widetilde{f}_{\alpha}^{\prime}+\widehat{f}_{\alpha}^{\prime}$ are decreasing (nonincreasing) for all $\alpha \in[0,1]$ on $] a, b[$;

2. If the second order derivatives $\widehat{f}_{\alpha}^{\prime \prime}$ and $\tilde{f}_{\alpha}^{\prime \prime}$ exist and are continuous, then:

(2-a) $F$ is $\left(\precsim_{L U}\right)$-convex on $] a, b\left[\right.$ if and only if $\widehat{f}_{\alpha}^{\prime \prime} \geq 0, \widehat{f}_{\alpha}^{\prime \prime}-\tilde{f}_{\alpha}^{\prime \prime} \geq 0$ and $\tilde{f}_{\alpha}^{\prime \prime}+\widehat{f}_{\alpha}^{\prime \prime} \geq 0$ for all $\alpha \in[0,1]$ on $] a, b[$;

(2-b) $F$ is $(\precsim L U)$-concave on $] a, b\left[\right.$ if and only if $\widehat{f}_{\alpha}^{\prime \prime} \leq 0, \widehat{f}_{\alpha}^{\prime \prime}-\widetilde{f}_{\alpha}^{\prime \prime} \leq 0$ and $\widetilde{f}_{\alpha}^{\prime \prime}+\widehat{f}_{\alpha}^{\prime \prime} \leq 0$ for all $\alpha \in[0,1]$ on $] a, b[$.

In Fig. 6 we picture the fuzzy-valued second order LgH-derivative of a function $F(x)$ and in Fig. 7 we show graphically the membership functions of $F_{L q H}^{\prime \prime}(x)$ at two points $x=2.2$ and $x=4.5$. Observe from Fig. 7 that $F_{L g H}^{\prime}(2.2)$ and $F_{L g H}^{\prime}(4.5)$ are not positive nor negative in the $\left(\precsim_{L U}\right)$ order, denoting that $F(x)$ is (locally) not convex nor concave around $x=2.2$ or $x=4.5$. See also Fig. 6 where regions of positive and negative second-order derivative can be identified.

Remark 1. Analogously to the relationship between the sign of second derivative and convexity for ordinary functions, we can establish conditions for convexity of fuzzy functions and the sign of the second order $\operatorname{LgH}$-derivative $F_{L g H}^{\prime \prime}(x)$; for example, a sufficient condition for strong $\prec_{L U}$-convexity is the following (compare with Proposition 10):

1. If $F_{L g H}^{\prime \prime}\left(x_{0}\right) \prec_{L U} 0$ then $F(x)$ is strongly concave at $x_{0}$.

2. If $F_{L g H}^{\prime \prime}\left(x_{0}\right) \succ_{L U} 0$ then $F(x)$ is strongly convex at $x_{0}$. 


\section{An Outline of Periodic Fuzzy-Valued Functions}

Definition 6. A function $F:[a, b] \rightarrow \mathbb{R}_{F}$ is said to be periodic if, for some nonzero constant $T \in] 0, b-a[$, it occurs that $F(x+T)=F(x)$ for all $x \in[a, b]$ with $x+T \in[a, b]$ (i.e., for all $x \in[a, b-T]$ ). A nonzero constant $T$ for which this is verified, is called a period of the function and if there exists a least positive constant $T$ with this property, it is called the fundamental period.

Obviously, if $F$ has a period $T$, then this also implies that $F_{\alpha}$ for all $\alpha \in[0,1]$ has a period $T$ i.e., for all $\alpha \in[0,1], \widehat{f_{\alpha}}$ and $\widetilde{f_{\alpha}}$ are periodic with period $T$. On the other hand, the periodicity of functions $F_{\alpha}$ for all $\alpha \in[0,1]$ does not necessarily imply the periodicity of $F$.

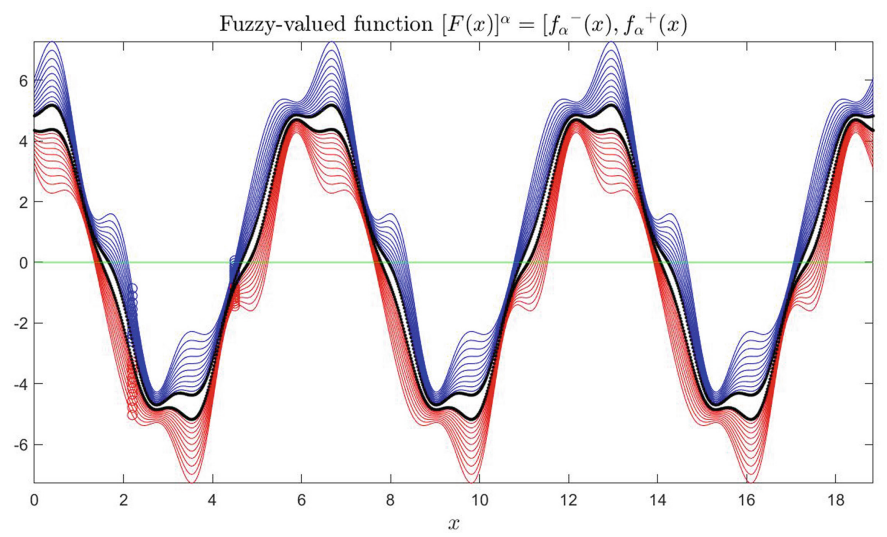

Fig. 8. Level-wise endpoint graphical representation of the periodic fuzzy-valued function $[F(x)]_{\alpha}=\left[\widehat{f}_{\alpha}(x)-\widetilde{f}_{\alpha}(x), \widehat{f}_{\alpha}(x)+\widetilde{f}_{\alpha}(x)\right]$ where $\widehat{f}_{\alpha}(x)=5 \cos (x)-(\sqrt{2}-1) \cos (5 x)$ and $\widetilde{f}_{\alpha}(x)=(1-0.6 \alpha)^{2}(1.5+\sin (4 x))$ for $x \in[0,6 \pi]$. The period is $p=2 \pi$.

Proposition 11. Let $F:[a, b] \rightarrow \mathbb{R}_{F}$ be a continuous function such that $[F(x)]_{\alpha}=\left(\widehat{f_{\alpha}}(x) ; \widetilde{f_{\alpha}}(x)\right)$ for all $\alpha \in[0,1]$ with $\widehat{f_{\alpha}}$ periodic of period $\widehat{T}$ and $\widetilde{f}_{\alpha}$ of period $\widetilde{T}$. Then it holds that:

(1) if the periods $\widehat{T}$ and $\widetilde{T}$ are commensurable, i.e., $\frac{\widehat{T}}{\widetilde{T}} \in \mathbb{Q}\left(\frac{\widehat{T}}{\widetilde{T}}=\frac{p}{q}\right.$, such that $p$ and $q$ are coprime) then the function $F$ is periodic of period $T=l c m(\widehat{T}, \widetilde{T})$, i.e,. $T$ is the least common multiple between $\widehat{T}$ and $\widetilde{T}$ (i.e., $T=p \widetilde{T}=q \widehat{T}$ );

(2) if the periods $\widehat{T}$ and $\widetilde{T}$ are not commensurable, i.e., $\frac{\widehat{T}}{\widetilde{T}} \notin \mathbb{Q}$, then function $F$ is not periodic.

A periodic function of period $p=2 \pi$ is given in Fig. 8. It is the function reported in all figures above, extended to the domain $[0,6 \pi]$. 


\section{Conclusions and Further Work}

We have developed new results to define monotonicity ans convexity or concavity for fuzzy-valued functions, in terms of the LU-partial order; similar results can be obtained for other types of partial orders. It appears that midpoint representation of the $\alpha$-cuts is a useful tool to analyse and visualize properties of fuzzy-valued functions. In further work, we will analyse important concepts of (local) minimal and maximal points for fuzzy valued functions, by the use of first-order and second-order LgH-derivatives.

\section{References}

1. Alefeld, G., Mayer, G.: Interval analysis: theory and applications. J. Comput. Appl. Math. 121, 421-464 (2000)

2. Kulpa, K.: Diagrammatic analysis of interval linear equations, Part I: basic notions and one-dimensional case. Reliable Comput. 9, 1-20 (2003)

3. Moore, R.E., Kearfott, R.B., Cloud, J.M.: Introduction to Interval Analysis. SIAM, Philadelphia (2009)

4. Stefanini, L.: A generalization of Hukuhara difference and division for interval and fuzzy arithmetic. Fuzzy Sets Syst. 161, 1564-1584 (2010)

5. Stefanini, L., Bede, B.: Generalized Hukuhara differentiability of interval-valued functions and interval differential equations. Nonlinear Anal. 71, 1311-1328 (2009)

6. Bede, B., Stefanini, L.: Generalized differentiability of fuzzy-valued functions. Fuzzy Sets Syst. 230, 119-141 (2013)

7. Stefanini, L., Arana-Jimenez, M.: Karush-Kuhn-Tucker conditions for interval and fuzzy optimization in several variables under total and directional generalized differentiability. Fuzzy Sets Syst. 362, 1-34 (2019)

8. Stefanini, L., Bede, B.: Generalized fuzzy differentiability with LU-parametric representations. Fuzzy Sets Syst. 257, 184-203 (2014)

9. Bede, B.: Mathematics of Fuzzy Sets and Fuzzy Logic. STUDFUZZ, vol. 295. Springer, Heidelberg (2013). https://doi.org/10.1007/978-3-642-35221-8

10. Stefanini, L., Bede, B.: A new gH-difference for multi-dimensional convex sets and convex fuzzy sets. Axioms 8, 48 (2019). https://doi.org/10.3390/axioms8020048

11. Guerra, M.L., Stefanini, L.: A comparison index for interval ordering based on generalized Hukuhara difference. Soft Comput. 16, 1931-1943 (2012)

12. Stefanini, L., Guerra, M.L., Amicizia, B.: Interval analysis and calculus for intervalvalued functions of a single variable. Part I: partial orders, gH-derivative, monotonicity. Axioms 8, 113 (2019). https://doi.org/10.3390/axioms8040113

13. Stefanini, L., Sorini, L., Amicizia, B.: Interval analysis and calculus for intervalvalued functions of a single variable. Part II: external points, convexity, periodicity. Axioms 8, 114 (2019). https://doi.org/10.3390/axioms8040114 